\title{
Adapting Cognitive Behavioral Therapy for Children and Adolescents with Complex Symptoms of Neurodevelopmental Disorders and Conduct Disorders
}

\section{Choo $\mathrm{C}^{*}$}

School of Health Professions, Murdoch University, 90 South St, WA 6150, Australia

"Corresponding author: Dr. Choo C, School of Health Professions, Murdoch University, 90 South St, WA 6150, Australia,. Tel +61 893602712 ; E-mail: c.choo@murdoch.edu.au

Received: June 03 2014; Accepted: July 02 2014; Published: July 152014

Copyright: (๑) 2014 Choo C, This is an open-access article distributed under the terms of the Creative Commons Attribution License, which permits unrestricted use, distribution, and reproduction in any medium, provided the original author and source are credited.

\begin{abstract}
Cognitive behavioral therapy (CBT) describes a group of psychotherapeutic techniques in which psychological distress and maladaptive behaviors are treated by changing cognitions and behaviors. Literature provides support for CBT as an effective intervention for children and adolescents, however, there is limited literature on how CBT can be adapted for children and adolescents with Neurodevelopmental Disorders and Conduct Disorders. This paper reviews relevant literature, and comments on how CBT can be adapted for children and adolescents with complex symptoms of Neurodevelopmental Disorders and Conduct Disorders, with a discussion of practical applications.
\end{abstract}

Keywords: Cognitive behavioral therapy; Children and adolescents; Neurodevelopmental Disorders; Conduct Disorders

\section{Introduction}

The term cognitive behavioural therapy describes a group of psychotherapeutic techniques in which psychological distress and maladaptive behaviours are treated by changing cognitions and behaviours [1]. CBT describes a hybrid of strategies to facilitate cognitive, behavioural, emotional and social change. Examples of interventions include the teaching of social skills through role play, problem solving techniques, coping skills, examining alternative ways of perception, and engagement in verbally mediated self-control.

The merging of behavioural and cognitive therapy into cognitive behavioural therapy (CBT) occurred in the 1980s in both Europe and North America. For children and adolescents, the process happened later [1,2]. Cognitive behavioural strategies with children and adolescents use enactive, performance-based procedures as well as cognitive interventions to produce changes in thinking, feeling and behavior [3] Cognitive interventions typically involve examining individual appraisal and beliefs about events, and then finding alternatives, with the aim to alter the resulting feeling and behaviour. When working with children, cognitive development should be considered, and adaptations made in pacing the content and speed of therapy, bearing in mind the younger child's limitations in metacognition and aptitude in labeling feelings. With younger children, the clinician is likely to be more active and will make use of a higher proportion of behavioural, as compared to cognitive strategies [2].

Literature provides support for CBT as an efficacious intervention for children and adolescents with diverse conditions such as eating disorder, social phobia, anxiety, aggression and other anger related problems [4-10].

\section{CBT in Practice}

The decision to use CBT with a child should be made after a clinical assessment which gathers information about a child's emotions, behaviours, relationships, family, school, and friendships [1,11]. This may provide the clinician with examples of the way the child thinks and feels.

CBT tends to be delivered in a series of sessions, usually between 8 and 12, with increasing intervals between the sessions to allow new techniques to be practiced or explored [1]. Sessions are tailored to the child's ability and may be short. Sessions usually begin with a setting of agenda around a mutually agreed problem list, the clinician may then move on to review homework tasks, examine thoughts and feelings about recent events, or learn and practice new cognitive or behavioural strategies. The technique chosen will take into consideration the child's capacity for memory, literacy, self-evaluation, empathy and self-control.

The sessions may include the usage of frequent summaries with feedback from the child; diagrams in thinking and communicating formulations; emotional recognition by self-monitoring to make links between events, mood and thoughts; activity scheduling, affectenhancing activities for self-reinforcers, cognitive restructuring, and coping skills training [2]. Other strategies include: coping self-talk, reframing of problems and role plays [3]. Some approaches are offered to the child in the form of experiments to encourage the child to try new strategies, or to encourage them to use 'self-statements' such as "this is bit scary but it's okay" [1].

\section{Engaging the child}

It is important that clinicians engage the client and create an active collaborative working alliance, to enable them to implement the therapy effectively [1]. In CBT, the explicit aims, agendas and collaborative approach may help the initial engagement. The clinician may use the child's feeling and thought about being brought in to the session to commence explorations of their perceptions of themselves 
and their world. The process of CBT models a facilitating relationship with an adult which may be particularly important for children who have previously had poor quality relationships with adults.

Attractive published materials may be used [12] individual tailormade charts may also be prepared. The clinician also has a role to increase the child's motivation, for example by using supportive phrases like "well done, even though it was difficult, I can see how hard you tried" [2].

\section{Overcoming developmental limitations}

$\mathrm{CBT}$ requires a minimum level of cognitive skills and is most suitable for those in mid-childhood or older [1]. CBT requires linguistic and cognitive skills to assess situations, and verbal reasoning and verbal memory to choose the most appropriate cause of action. CBT should take place in the context of a child's level of cognitive development. Clinicians need an understanding of the child's psychological and cognitive functioning. In general, children acquire a more mature style of self-control, self-evaluation, and emotional recognition in middle childhood [13]. While a bright well-motivated adolescent's grasp of unmodified adult-type CBT programmes can be rewarding, younger children may find programmes such as thought diaries beyond them, unless they are suitably modified. Many techniques involve the use of written materials such as information sheets and worksheets. These need to be adapted to the child's level of cognitive development. Before therapy properly begins, children may benefit from emotional education, during which they learn to distinguish different emotional states and link emotions with thoughts and events $[1,2]$. The usage of developmentally appropriate tasks will encourage healthy cognitive development of the child. However, developmental limitations remain for children in working with higherorder abstractions such as reflecting on hypotheses and evaluating evidence for and against a belief, which may not develop till middle adolescence [13].

Mental handicap does not preclude the use of CBT, in fact CBT techniques have been found to be effective in anger control for people with learning disabilities [14]. Non-cognitive procedures of relaxation and monitoring have been found to be particularly effective with challenging behaviors. For children with learning disabilities, the language used could be modified, the training could be done over a longer period, and the self-instruction could be narrowed down to a general one such as "take it easy" to minimize the number of decisions to be made. Information could be presented visually to make abstract concepts more concrete, such as using a traffic light to prompt problem solving and using a thermometer to measure arousal, the clinician could also provide increased prompts to generate possible solutions, and use more contextualized and specific perspectives to discuss usage of specific CBT techniques.

\section{CBT as a Multi-modal approach}

It is important that the clinician is aware of the family structure, the systematic implications of any interventions, and psychosocial factors such as history of abuse or a learning disability, that may impact on assessment and therapy with children and adolescents [11]. It is often useful to include support from the school, and to engage the parents. Parent training can reinforce therapeutic achievement for the children. Parents can be educated in the CBT techniques used with their child and encouraged to offer positive feedback to their child when they attempt to try it at home [1]. With a broad based CBT approach, a psycho-educational element of information-giving by discussions, supplemented by fact sheets is important. This is likely to increase compliance and is often appreciated by the child, family, school, and the referrer [2].

Adaptations of CBT for Conduct Disorder, Attention-Deficit Hyperactivity Disorder (ADHD) and aggression

In my therapeutic work with children and adolescents, I have made adaptations of CBT for different individuals, as the content of their cognition is different. Anger may give rise to personal rules such as "it is not fair", while anxiety may give rise to Catastrophizing, for example "it will be a disaster". For clients with cognitive deficits and deficits of social skills and problem-solving, which is often concurrent with ADHD and conduct disorder [15], I have adapted the application of CBT techniques to suit the needs of these clients with complex comorbid symptoms.

\section{Conduct disorder}

For conduct disorder, CBT is useful as part of a multi-modal approach with involvement from the parents and the school [11]. Parents may need specific instructions in management techniques, for example in using positive reinforcement for compliance with a child with conduct disorder [2]. Operant techniques rewarding pro-social behaviors and discouraging antisocial behaviors are supplemented by instructions, discussion, modeling strategies, rehearsal, prompting and feedback. Social skills training is often used, and may include: emotional education, self-monitoring of feelings and behaviors, selfinstructions, self-reinforcement, social perspective taking, using vignettes to enable the child to understand the intentions of others in social situations; and problem-solving.

My experience with children and adolescents with conduct disorder is that they tend to attribute hostility to others and underestimate their own aggression in any conflict. They value aggression as effective in problem solving and enhancing their self-esteem. Examples of effective CBT programmes for these clients include anger management programmes to help them identify their aggressive behavior and the conditions that provoke and maintain it, and to reinforce the use of effective strategies to manage their anger [2].

\section{Attention Deficit Hyperactivity Disorder (ADHD)}

Children with ADHD often show cognitive deficits including deficiencies in overall self-regulation, difficulties with sophisticated problem solving, and poor adaptation of social behavior [15]. Although research shows that CBT may be effective in moderating impulsivity, the impact of CBT on restlessness and distractibility has not been firmly established [2,3].

CBT techniques such as reinforcement and problem solving skills have been reported to be used, together with emphasis on the role of parents and family in program implementation, but outcome studies have not been optimistic when compared with medications [3].

\section{Aggression}

A meta-analysis showed that CBT is an effective treatment for anger related problems in children and adolescents [10]. CBT has been shown to be effective for boys with aggression and disruptive behaviors in raising their self-esteem, and improving their social problem solving skills, with lower rate of disruption and aggression in the classroom [8]. The outcome study was done on a school based 
group programmer for aggressive boys (aged 10 to 12 years old) which was held over 12 to 18 sessions in 4-5 months. The programme included: establishing group rules, using self-statements, discussion of scenarios, and practicing social problem solving skills with discussions, role plays and dialogue. The outcome was positive 3 years after the intervention for the subset of boys who had booster sessions.

My experience with aggressive children is they often show many cognitive deficits and distortions such as the inaccurate labeling of affect, deficient problem solving ability, the ready perception of hostile intentions in the behavior of others, and insufficient forethought prior to action. Programmes using CBT techniques for training in problem solving and anger control is beneficial. Many anger management programmes use techniques in relaxation, self-monitoring of behavior or feelings, recognition of triggers and self-inhibitory statements such as "stop, think, do" to slow down the automaticity of their response. Other techniques include psycho-education about anger and other emotions, self-instruction, and social problem solving with emphasis on verbal assertion and compromise solutions $[3,14]$. A meta-analysis of CBT for anger related problems showed that skills development and multi-modal treatments that teach actual behaviors are more effective than treatment to modify internal constructs, and problem solving treatments showed greater effects for reducing subjective reports of anger [10]. Social skills training is another common approach for anger control treatment for children [16] with techniques derived from learning theory [17] including skills acquisition, and reduction of interfering behaviours [18].

\section{Conclusion}

In conclusion, this paper supports existing literature on the usefulness of CBT with children and adolescents. It contributes to literature by commenting on how CBT can be adapted for use with children and adolescents as a multi-modal approach, for wide ranging problems such as learning difficulties, Conduct Disorder, AttentionDeficit Hyperactivity Disorder (ADHD) and aggression. Personally, I have adapted CBT in both individual and group programmes for clients with complex co-morbid, and wide ranging problems such as learning difficulties, Conduct Disorder, Attention-Deficit Hyperactivity Disorder (ADHD) and aggression in my work with children and adolescents from diverse Asian and Western cultures, in diverse settings over the past 10 years. There have been encouraging outcomes reported by clients, parents, schools and other referrers. It is anticipated that these outcomes will be reported in a series of case studies.

\section{References}

1. Kaplan CA, Thompson AE, Searson SM (1995) Cognitive behaviour therapy in children and adolescents. Arch Dis Child 73: 472-475.
2. Bailey V (2001) Cognitive - behavioural therapies for children and adolescents. Advances in Psychiatric Treatment 7: 224-232.

3. Kendall PC (1993) Cognitive-behavioral therapies with youth: guiding theory, current status, and emerging developments. J Consult Clin Psychol 61: 235-247.

4. Lock J (2005) Adjusting cognitive behavior therapy for adolescents with bulimia nervosa: results of case series. Am J Psychother 59: 267-281.

5. Lock J, Fitzpatrick K (2007) Evidence-based treatment for children and adolescents with eating disorders: Family therapy and family facilitated cognitive behavioral therapy. Journal of Contemporary Psychotherapy 37: $145-155$.

6. Melfsen S, Kuhnemind M, Schwieger J, Warnke A et al. (2011) Cognitive behavioural therapy of socially phobic children focusing on cognition: A randomised wait-list control study. Child Adolesc Psychiatry Ment Health 5: 1-12.

7. Shortt AL, Barrett PM, Fox TL (2001) Evaluating the FRIENDS program: a cognitive-behavioral group treatment for anxious children and their parents. J Clin Child Psychol 30: 525-535.

8. Lochman JE (1992) Cognitive-behavioral intervention with aggressive boys: three-year follow-up and preventive effects. J Consult Clin Psychol 60: 426-432.

9. Gansle K (2005) The effectiveness of school-based anger interventions and programs: A meta- analysis. Journal of School Psychology 43: 321-341.

10. Whitaker S (2001) Anger control for people with learning disabilities: A critical review. Behavioral and Cognitive Psychotherapy 29: 277-293.

11. Carr A (2006) The handbook of child and adolescent clinical psychology (2nd edition), Routledge, East Sussex.

12. Seiler L (2008) Cool Connections with Cognitive Behavioural Therapy. Jessica Kingsley Publishers, Great Britan.

13. Santrock J (2008) Life-span Development, McGraw-Hill, New York.

14. Sukhodolsky D, Kassinove H, Groman B (2004) Cognitive behavioural therapy for anger in children and adolescents: A meta analysis. Aggression and violent behavior, 9: 247-269.

15. American Psychiatric Association (2013). Diagnostic and statistical manual of mental disorders (5th edition).

16. Stern JB, Fodor IG (1989) Anger control in children: A review of social skills and cognitive behavioral approaches to dealing with aggressive children. Child and Family Behavior Therapy 11: 1-20.

17. Frey KS, Hirschstein MK, Guzzo BA (2000) Second Step: Preventing aggression by promoting social competence. Journal of Emotional and Behavioral Disorders 8: 102-112.

18. Gresham FM (1988) Social skills: Conceptual and applied aspects of assessment, training, and social validation. In J. C. Witt, S. N. Elliott, \& F. M. Gresham (Eds.), Handbook of behavior therapy in education , Plenum, New York. 